\title{
Effect of Sleep Disturbance on Efficacy of Esketamine in Treatment-Resistant Depression: Findings from Randomized Controlled Trials
}

\author{
Stephane Borentain (1D) \\ David Williamson ${ }^{2,3}$ \\ Ibrahim Turkoz $\mathbb{D D}^{4}$ \\ Vanina Popova ${ }^{5}$ \\ William V McCall ${ }^{3}$ \\ Maju Mathews' \\ Frank Wiegand'
}

'Department of Global Medical Affairs, Janssen Research \& Development, LLC Titusville, NJ, USA; ${ }^{2}$ CNS Scientific Affairs Liaisons, Janssen Scientific Affairs, LLC, Titusville, NJ, USA; ${ }^{3}$ Department of Psychiatry and Health Behavior, Medical College of Georgia, Augusta University, Augusta, GA, USA; ${ }^{4}$ Department of Clinical Statistics, Janssen Research \& Development, LLC, Titusville, NJ, USA; ${ }^{5}$ Department of Neuroscience Clinical Development, Janssen Research \& Development, Beerse, Belgium
Correspondence: Stephane Borentain Department of Global Medical Affairs, Janssen Research \& Development, LLC, I 125 Trenton-Harbourton Road,

Titusville, NJ, 08560, USA

$\mathrm{Tel}+$ I 609-7304420

Email SBorentI@ITS.JNJ.com
Purpose: To evaluate the relationship of sleep disturbance to the antidepressant effects of esketamine.

Materials and Methods: Two double-blind, 4-week studies randomized adults with treatment-resistant depression (TRD) to placebo or esketamine nasal spray, each with newly initiated antidepressant. Sleep was assessed using Montgomery-Åsberg Depression Rating Scale (MADRS) item 4. Change in response ( $\geq 50 \%$ decrease in MADRS total score) and remission (total MADRS score $\leq 12$ ) at day 28 was examined by presence/absence of baseline sleep disturbance using logistic regression models. Impact on reported sleep disturbance (MADRS item 4 score) was examined using ANCOVA models.

Results: At baseline, most patients reported disturbed sleep - moderate/severe $(65.3 \%, 369$ / $565)$, mild $(25.3 \%, 143 / 565)$, or none/slightly $(9.4 \%, 53 / 565)$ - with similar distribution between treatment groups. A higher proportion of esketamine-treated patients achieved response $(\mathrm{OR}=2.05 ; 95 \% \mathrm{CI}: 1.40-3.02 ; P<0.001)$ and remission $(\mathrm{OR}=1.81 ; 95 \% \mathrm{CI}$ : 1.23-2.66; $P=0.003$ ) at day 28 compared to antidepressant plus placebo, regardless of presence/severity of sleep disturbance. Consistent with this, sleep (MADRS item 4 score) improved in both groups after the first dose, more so with esketamine by day 8 (betweengroup difference: $P \leq 0.02$ at all time points). Across both treatment groups, 1-point improvement in sleep at day 8 increased the probability of antidepressant response on day 28 by $26 \%(\mathrm{OR}=1.26,95 \% \mathrm{CI}: 1.12-1.42 ; P<0.001)$, and remission by $28 \%(\mathrm{OR}=1.28$, 95\% CI: $1.14-1.43 ; P<0.001)$.

Conclusion: Antidepressant efficacy of esketamine was demonstrated in patients with TRD, regardless of the presence of sleep disturbance. After 8 days of treatment and thereafter, significantly more esketamine-treated patients reported improvement in sleep versus antidepressant plus placebo.

Keywords: esketamine, treatment-resistant depression, sleep disturbance, insomnia

\section{Introduction}

The majority of patients with non-psychotic major depressive disorder (MDD) have symptoms of insomnia. ${ }^{1}$ In fact, sleep disturbance is a criterion for a DSM-5 diagnosis of MDD. ${ }^{2}$ Insomnia symptoms increase the risk for both new-onset and recurrent depression. ${ }^{3,4}$ Additionally, insomnia symptoms concurrent with MDD has been associated with prolonged duration of depressive episodes, greater symptom severity, treatment resistance, higher prevalence of other psychiatric disorders (eg, anxiety), increased rate of suicidal ideation/behavior, and impaired daytime functioning, work performance, and long-term absenteeism, leading to deterioration 
in quality-of-life. ${ }^{5-11}$ Correspondingly, resolution of sleep disturbance in MDD is associated with improvement in patient-reported quality of 1 ife, ${ }^{12}$ reduction in suicidal ideation, ${ }^{13}$ and improvement of depressive symptoms. ${ }^{14}$

Sleep disturbance has been reported to portend poorer efficacy with standard antidepressants and, when untreated, adversely affects long-term treatment outcomes in depressed patients. ${ }^{15-18}$ Severity of symptoms may also be a factor: Mason et al, ${ }^{19}$ using data from the Sequenced Treatment Alternatives to Relieve Depression (STAR*D) trial, reported that those patients with MDD who have more severe insomnia at baseline are less likely to achieve remission of depressive symptoms. Others have reported that baseline level of insomnia does not impact acute treatment (paroxetine, sertraline or fluoxetine) efficacy in MDD patients. ${ }^{20}$ In contrast to the findings from studies of standard antidepressants, Liu et $\mathrm{al}^{21}$ reported that patients with a high level of insomnia who received six intravenous infusions of ketamine over 12 days for depression were more likely to respond and to remit than those with a lower level of insomnia. Taken together, the varying relationships between sleep disturbance and antidepressant efficacy might depend on the mechanism of action of the antidepressant.

Esketamine, the S-enantiomer of ketamine, is approved by the US Food and Drug Administration, ${ }^{22}$ European Medicines Agency, ${ }^{23}$ and other health authorities, in combination with an oral antidepressant, for treatment-resistant depression (TRD) in adults. These approvals were based, in large measure, upon the pivotal TRANSFORM-2 study and the supportive TRANSFORM-1 study, ${ }^{24,25}$ in which greater improvement in depressive symptoms was observed among patients randomized to esketamine plus an antidepressant, compared to antidepressant plus placebo, after a month of treatment (LS mean change in Montgomery-Åsberg Depression Rating Scale [MADRS] [95\% confidence interval $[\mathrm{CI}]:-4.4[-6.6,-2.1])$.

To evaluate the relationship of sleep disturbance to the antidepressant effects of esketamine, and vice versa, we conducted post-hoc analyses of pooled data from these two similarly designed TRANSFORM studies of TRD. ${ }^{24,25}$ The aims of these post-hoc analyses were to determine: 1) if baseline severity of reported sleep disturbance moderates antidepressant response; 2) if the presence and severity of reported sleep disturbance change as a function of treatment over the first month; and, 3) if early change in reported sleep disturbance severity is related to likelihood of response or remission of depressive symptoms at day 28 .

\section{Materials and Methods Study Design}

TRANSFORM-1 and TRANSFORM-2 were similarly designed, 4-week, double-blind, randomized, activecontrolled, multicenter trials of esketamine nasal spray in outpatients with TRD. ${ }^{24,25}$ The trials comprised a 4-week screening phase during which treatment response to the current antidepressant(s) was prospectively assessed, a 4-week double-blind treatment phase, and a (up to 24weeks) post-treatment follow-up phase assessing safety.

Both studies were approved by independent review boards/ethics committees (names provided in Table S1), both studies were conducted in accordance with the ethical principles that have their origin in the Declaration of Helsinki, and written informed consent was obtained from all patients before their study participation (clinical trials.gov identifiers: NCT02417064; NCT02418585).

\section{Patients}

Both TRANSFORM studies enrolled adults (18-64 years) with recurrent MDD (per Diagnostic and Statistical Manual of Mental Disorders, Fifth Edition [DSM-5] criteria $^{2}$ ), without psychotic features, and a total score $\geq 34$ on the clinician-rated Inventory of Depressive Symptomatology. ${ }^{26}$ At randomization, eligible participants had TRD defined as non-response to an adequate (dose and duration) course of at least two oral antidepressants during the current depressive episode. Key exclusion criteria included moderate-to-severe substance or alcohol use disorder within the prior 6 months, positive test result(s) for specified drugs of abuse, and use of high-dose benzodiazepines (ie, total daily dose equivalent $>6 \mathrm{mg}$ of lorazepam). Full lists of the inclusion and exclusion criteria for each study are published. ${ }^{24,25}$

At the end of the screening phase, nonresponders to their ongoing oral antidepressant discontinued all current antidepressant treatment(s) and were randomized to double-blind treatment with twice-weekly esketamine nasal spray or matching placebo nasal spray for 4 weeks, each in combination with a newly initiated, open-label oral antidepressant taken daily. Patients received either a 56 or $84 \mathrm{mg}$ dose of esketamine nasal spray - fixed dose in TRANSFORM-1 and flexible dose in TRANSFORM-2 (ie, $56 \mathrm{mg}$ on day 1 , then either $56 \mathrm{mg}$ or $84 \mathrm{mg}$ on days 
$4,8,11$, or 15 based on efficacy and tolerability, after which the dose remained stable).

The investigator selected the oral antidepressant from two different drug classes: a selective serotonin reuptake inhibitor (SSRI; escitalopram or sertraline) or a serotoninnorepinephrine reuptake inhibitor (SNRI; duloxetine or venlafaxine extended release). Benzodiazepines (at dose equivalent $\leq 6 \mathrm{mg}$ lorazepam) and non-benzodiazepine sleep medications (eg, zolpidem, zaleplon, eszopiclone, ramelteon) taken prior to study enrollment could be continued during double-blind treatment, except in the 12 hours prior to dosing of study drug. Concomitant use of lithium or antipsychotic medications was prohibited.

\section{Assessments}

Independent, blinded, remote raters performed MADRS assessments, via structured interview, ${ }^{27,28}$ at baseline (7-day recall period) and all post-baseline visits during the double-blind treatment phase (24-hour recall period at day 2; 7-day recall period at all subsequent visits). If performed on the day of the scheduled clinic visit for an intranasal treatment session, the MADRS assessment was performed prior to the intranasal treatment session.

Treatment-emergent adverse events were appraised throughout the study.

\section{Statistical Analyses}

Data from both studies were pooled and analyses included all randomized patients who received at least 1 dose of intranasal study drug and 1 dose of oral antidepressant. Analyses of efficacy and safety endpoints were evaluated in groups of patients according to the level of sleep disturbance at baseline. Sleep disturbance was evaluated using MADRS item 4, assessing perceived reduction in duration or depth of sleep versus patient's normal pattern when well. In the analyses, we defined sleep disturbance as absent/slight (scores of $0-1$ ), mild (scores of 2-3), or moderate/severe (scores of 4-6; sleep reduced or broken by $\geq 2$ hours to $<2-3$ hours of sleep). ${ }^{29,30}$

Between-group differences for baseline demographics and disease characteristics were analyzed by $t$-test for continuous variables and by chi-square test for categorical variables.

Utilizing the approach taken by other research groups to evaluate the impact of antidepressant on sleep disturbance, ${ }^{31-33}$ the between-group difference on change from baseline for reported sleep disturbance (ie, MADRS item 4 score) was examined using an analysis of covariance (ANCOVA) models with fixed effects for treatment, study, region, class of oral antidepressant (SNRI or SSRI), and baseline value as a covariate. We defined clinically meaningful improvement in sleep disturbance as $\geq 2$-point decrease in MADRS item 4 score from baseline, ${ }^{29,30}$ and compared this variable between treatment groups using the Cochran-Mantel-Haenszel (CMH) test controlling for region, class of oral antidepressant, and study.

Multiple logistic regression models were used to examine whether observed treatment difference at day 28 for antidepressant response (defined as $\geq 50 \%$ decrease in MADRS total score from baseline) and for remission (defined as MADRS total score $\leq 12$ ) ) $^{32,34}$ were dependent on baseline sleep disturbance. The probability of response and probability of remission were computed between treatment groups by 1-point increase in baseline MADRS item 4 score, and odds ratios (OR) and 95\% CIs were calculated. Sleep disturbance changes by week 1 (day 8) were also quantified and regressed against the mean MADRS total score changes, and separately on response and remission rates at day 28. Separate models included interaction term between treatment and change in sleep disturbance level at day 8 .

Adverse events were summarized descriptively.

\section{Results}

Overall, 573 patients were randomized to double-blind treatment (Figure 1); of these, 8 patients did not receive any study drug. Thus, the data set for the analyses included 565 patients (343 in the esketamine plus antidepressant group and 222 in the antidepressant plus placebo group). The discontinuation rate was low across all the baseline sleep disturbance status and treatment subgroups: 1 (3.1\%), $11(13.8 \%)$, and $25(10.8 \%)$ esketamine-treated patients and $2(9.5 \%), 5(7.9 \%)$, and $9(6.5 \%)$ placebotreated patients with absent/slight, mild, and moderate/ severe sleep disturbance at baseline, respectively, withdrew from the study prematurely.

At baseline, most patients reported disturbed sleep, approximately two-thirds $(369,65.3 \%)$ having moderate/ severe disturbance, with distributions similar between treatment groups within each sleep disturbance subgroup (Table 1). The sleep disturbance subgroups were similar with respect to demographic and most disease characteristics (Table 1). Mean duration of the current depressive episode ranged from 147.2 to 192.3 weeks across the subgroups by sleep disturbance status and treatment 
Screening/Prospective Observational Phase (4 weeks)
Double-Blind Treatment Phase

(4 weeks)

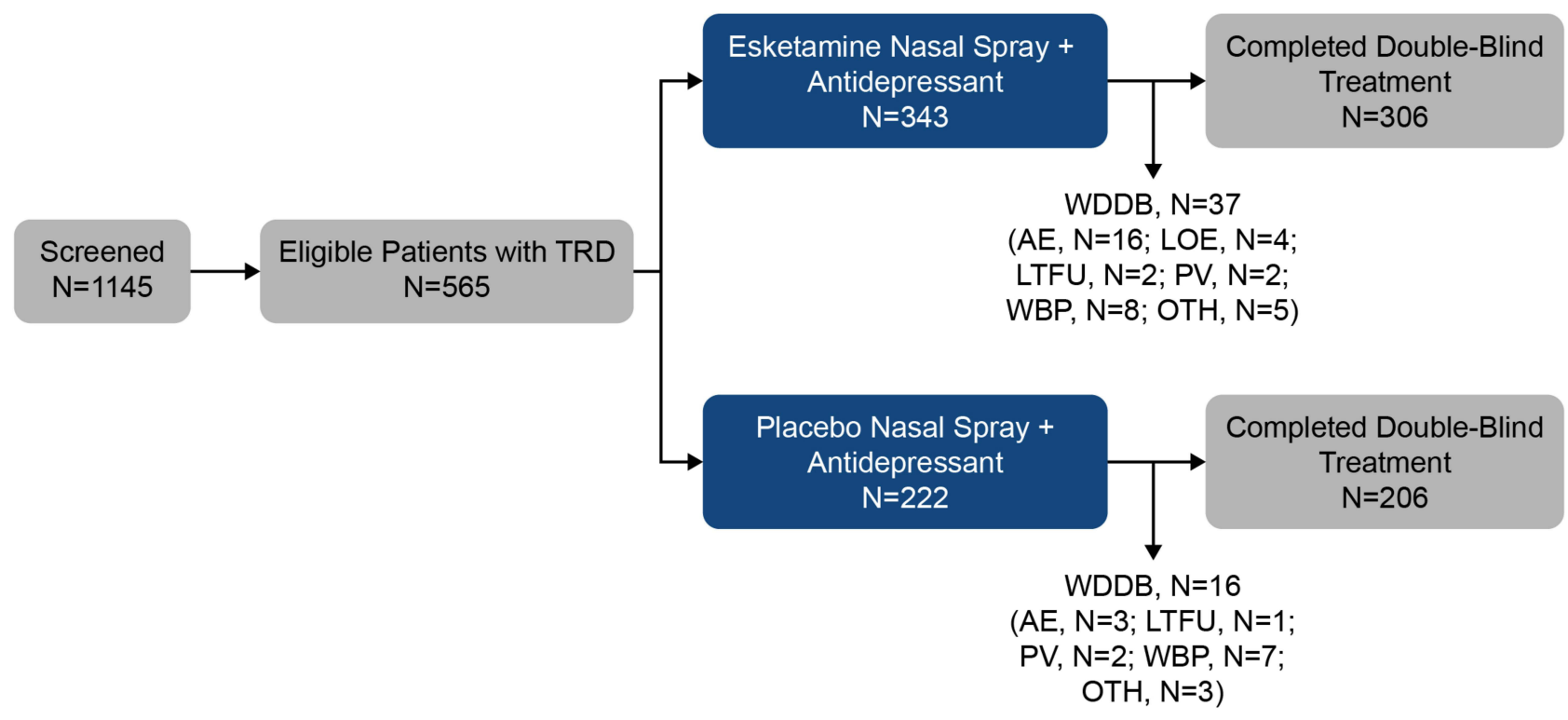

Figure I Disposition of Patients.

Notes: One (3.1\%), II (13.8\%), and 25 (10.8\%) esketamine-treated patients and 2 ( $9.5 \%), 5$ (7.9\%), and 9 (6.5\%) placebo-treated patients with absent/slight, mild, and moderate/severe sleep disturbance at baseline, respectively, withdrew from the study prematurely.

Abbreviations: AE, adverse event; LOE, lack of efficacy; LTFU, lost to follow-up; N, number of patients; OTH, other reason for withdrawal; PV, protocol violation; TRD, treatment-resistant depression; WBP, withdrawal by patient; WDDB, withdrawal from double-blind phase.

group. Baseline depression severity (as quantified by MADRS total score, excluding item 4) was consistent between groups within each stratum of sleep disturbance; however, at baseline, patients with more severe depression also had more severe reported sleep disturbance: mean MADRS total - excluding item 4 - score was higher in the subgroups with greater sleep disturbance (32.0 for patients with MADRS item 4 score of 2 or $3[P=0.488]$ and 34.0 for patients with MADRS item 4 score of 4,5 , or $6[P<0.001]$ versus 31.0 for patients with MADRS item 4 score of 0 or 1 ). Within each stratum of sleep disturbance, the distribution of oral antidepressant used was similar between treatment groups, whereas stable benzodiazepine usage at randomization was more common among patients in the esketamine plus antidepressant group versus the antidepressant plus placebo group.

\section{Impact of Baseline Reported Sleep Disturbance}

The more severe sleep disturbance was reported to be at baseline, the greater the likelihood that depressive symptoms would respond or remit by day 28 , as indicated by the results of both univariate and multiple logistic regression analyses. Across treatment groups, a 1-point increase (ie, more severe) in reported sleep disturbance at baseline was associated with a $23 \%$ greater probability of antidepressant response $(\mathrm{OR}=1.23,95 \% \mathrm{CI}$ : 1.08 , $1.40 ; P=0.001)$ and $5.0 \%$ greater probability of remission $(\mathrm{OR}=1.05,95 \% \mathrm{CI}: 0.93,1.20 ; P=0.437)$.

A higher proportion of esketamine-treated patients achieved response at day 28 compared to antidepressant plus placebo $(\mathrm{OR}=2.05$; 95\% CI: $1.40,3.02 ; P<0.001)$, regardless of presence/severity of sleep disturbance (Figure 2A). A similar trend was observed for remission rate $(\mathrm{OR}=1.81 ; 95 \% \mathrm{CI}: 1.23,2.66 ; P=0.003)$ (Figure 2B).

\section{Does the presence and severity of reported sleep disturbance change as a function of treatment?}

Sleep improved in both treatment groups, beginning 24 hours after the first dose (ie, day 2; Figure 3). The sleep of patients treated with esketamine plus antidepressant improved more than that of patients treated with antidepressant plus placebo on day 8 (between-group mean 
Table I Baseline Demographics and Disease Characteristics by Status of Sleep Disturbance Among Patients with Treatment-Resistant Depression

\begin{tabular}{|c|c|c|c|c|c|c|}
\hline \multirow[t]{4}{*}{ Parameter } & \multicolumn{6}{|c|}{ Baseline MADRS Item 4 Sleep Score } \\
\hline & \multicolumn{2}{|c|}{0 or $\mathbf{I}$} & \multicolumn{2}{|c|}{2 or 3} & \multicolumn{2}{|c|}{4,5 , or 6} \\
\hline & $\begin{array}{c}\text { Esketamine + } \\
\text { Antidepressant }\end{array}$ & $\begin{array}{l}\text { Antidepressant } \\
\quad+\text { Placebo }\end{array}$ & $\begin{array}{l}\text { Esketamine + } \\
\text { Antidepressant }\end{array}$ & $\begin{array}{l}\text { Antidepressant } \\
+ \text { Placebo }\end{array}$ & $\begin{array}{c}\text { Esketamine + } \\
\text { Antidepressant }\end{array}$ & $\begin{array}{l}\text { Antidepressant } \\
\quad+\text { Placebo }\end{array}$ \\
\hline & $\mathrm{n}=\mathbf{3 2}$ & $\mathbf{n}=\mathbf{2 1}$ & $\mathrm{n}=\mathbf{8 0 ^ { \mathrm { a } }}$ & $n=63^{b}$ & $n=23 I^{c}$ & $n=138^{d}$ \\
\hline Mean age, years (SD) & $46.7(11.63)$ & $48.2(12.93)$ & $44.4(12.79)$ & $47.5(11.78)$ & $46.0(11.20)$ & $46.0(10.73)$ \\
\hline \multicolumn{7}{|l|}{ Sex, n (\%) } \\
\hline Male & $13(40.6)$ & $7(33.3)$ & $24(30.0)$ & $29(46.0)$ & $71(30.7)$ & $42(30.4)$ \\
\hline Female & $19(59.4)$ & $14(66.7)$ & $56(70.0)$ & $34(54.0)$ & $160(69.3)$ & $96(69.6)$ \\
\hline \multicolumn{7}{|l|}{ Race, n (\%) } \\
\hline White & $27(84.4)$ & $20(95.2)$ & $70(93.3)$ & $55(88.7)$ & $185(84.1)$ & $113(86.9)$ \\
\hline Black/African American & I (3.I) & 0 & $2(2.7)$ & I ( $(1.6)$ & $17(7.7)$ & $9(6.9)$ \\
\hline Other & $4(12.5)$ & I (4.8) & $3(4.0)$ & $6(9.7)$ & $18(8.2)$ & $8(6.2)$ \\
\hline \multicolumn{7}{|l|}{ Region, n (\%) } \\
\hline Europe & $15(46.9)$ & $9(42.9)$ & $29(36.3)$ & $25(39.7)$ & $81(35.1)$ & $60(43.5)$ \\
\hline North America & II (34.4) & $7(33.3)$ & $37(46.3)$ & $31(49.2)$ & $101(43.7)$ & $57(4 \mid .3)$ \\
\hline Other & $6(18.8)$ & $5(23.8)$ & $14(17.5)$ & $7(11.1)$ & $49(21.2)$ & $21(15.2)$ \\
\hline $\begin{array}{l}\text { Mean duration of current episode, weeks } \\
\text { (SD) }\end{array}$ & $147.2(248.6)$ & $192.3(367.9)$ & $159.2(267.0)$ & $164.0(243.0)$ & $185.4(261.5)$ & $147.2(200.5)$ \\
\hline \multicolumn{7}{|l|}{ No. of previous antidepressants, $n(\%)^{e}$} \\
\hline I or 2 & $19(61.3)$ & $14(66.7)$ & $54(67.5)$ & $35(55.6)$ & $143(62.2)$ & $90(65.2)$ \\
\hline$\geq 3$ & $12(38.7)$ & $7(33.3)$ & $26(32.5)$ & $28(44.4)$ & $87(37.8)$ & $48(34.8)$ \\
\hline \multicolumn{7}{|l|}{ Oral antidepressant, n (\%) } \\
\hline Duloxetine & $14(43.8)$ & $10(47.6)$ & $28(35.0)$ & $31(49.2)$ & $110(47.6)$ & $64(46.4)$ \\
\hline Escitalopram & $8(25.0)$ & $3(14.3)$ & $12(15.0)$ & $9(14.3)$ & $50(21.7)$ & $29(21.0)$ \\
\hline Sertraline & $4(12.5)$ & $4(19.1)$ & $20(25.0)$ & $15(23.8)$ & $40(17.3)$ & $22(15.9)$ \\
\hline Venlafaxine $X R$ & $6(18.8)$ & $4(19.1)$ & $20(25.0)$ & $8(12.7)$ & $31(13.4)$ & $23(16.7)$ \\
\hline $\begin{array}{l}\text { Mean MADRS total score minus item } 4 \\
\text { (SD) }\end{array}$ & $32.0(4.98)$ & $31.4(4.82)$ & $32.5(4.91)$ & $32.0(4.70)$ & $34.4(4.73)$ & $34.8(5.43)$ \\
\hline Comorbid anxiety disorder, $\mathrm{n}(\%)$ & $20(64.5)$ & $12(57.1)$ & $54(67.5)$ & $42(66.7)$ & $172(74.8)$ & $102(74.5)$ \\
\hline MADRS item 4 score, mean (SD) & $0.5(0.5 \mathrm{I})$ & $0.7(0.48)$ & $2.6(0.48)$ & $2.6(0.50)$ & $4.6(0.58)$ & $4.6(0.58)$ \\
\hline Benzodiazepine use, $\mathrm{n}(\%)^{g, h}$ & $13(40.6)$ & $6(28.6)$ & $27(33.8)$ & $15(23.8)$ & $74(32.0)$ & $40(29.0)$ \\
\hline $\begin{array}{l}\text { Non-benzodiazepine sedative/hypnotic } \\
\text { use }^{\text {h,i }}, \text { n (\%) }\end{array}$ & $2(6.3)$ & I (4.8) & $5(6.3)$ & $5(7.9)$ & $14(6.1)$ & $10(7.3)$ \\
\hline
\end{tabular}

Notes: $P>0.05$ for all comparisons between treatment groups within each sleep disturbance sub-group; between treatment group comparisons were based on $t$-test for continuous variables or Chi-square test for categorical variables. ${ }^{\mathrm{a}} 5$ missing for race. ${ }^{\mathrm{b}} \mathrm{I}$ missing for race. ${ }^{\mathrm{c}} \mathrm{II}$ missing for race; I missing for number of previous antidepressants. ${ }^{\mathrm{d}} 8$ missing for race. ${ }^{\mathrm{e}}$ Number of antidepressant medications with non-response (defined as $\leq 25 \%$ improvement) taken for at least 6 weeks during the

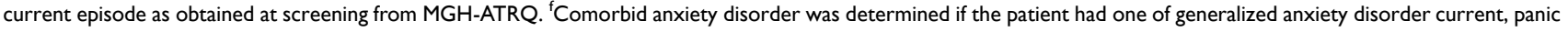
disorder current, social anxiety disorder current, posttraumatic stress disorder current or obsessive-compulsive disorder current by MINI or GAD-7 total score $\geq 10$ at screening and baseline. ${ }^{g}$ Sleep disturbance severity subgroup comparison within the same treatment group (esketamine [in bold], placebo) by Chi-square test: $P$ value for 0 or I vs 2 or $3(\mathbf{0 . 4 9 3}, 0.663)$; 0 or I vs $4-6(\mathbf{0 . 3 3 3}, 0.969)$. ' Excluding use on an as needed (prn) basis. 'Zaleplon, zolpidem, zolpidem tartrate, zopiclone.

Abbreviations: GAD-7, generalized anxiety disorder 7-item scale; MADRS, Montgomery-Asberg Depression Rating Scale; MGH-ATRQ, Massachusetts General Hospital Antidepressant Treatment Response Questionnaire; MINI, Mini-International Neuropsychiatric Interview; SD, standard deviation.

(SE) difference in MADRS item 4 score: $-0.4(0.12)$, $P=0.001)$ and at all subsequent evaluation timepoints through day 28 (mean (SE) between-group difference in MADRS item 4 score: $-0.3(0.15), P=0.020)$. In line with these findings, more patients in the esketamine plus antidepressant group achieved clinically meaningful improvement in sleep (which we defined as $\geq 2$-point decrease in MADRS item 4 score from baseline) than patients in the antidepressant plus placebo group at day 8 (26.1\% [86/330] and 15.7\%, [34/216], respectively, 
A Response

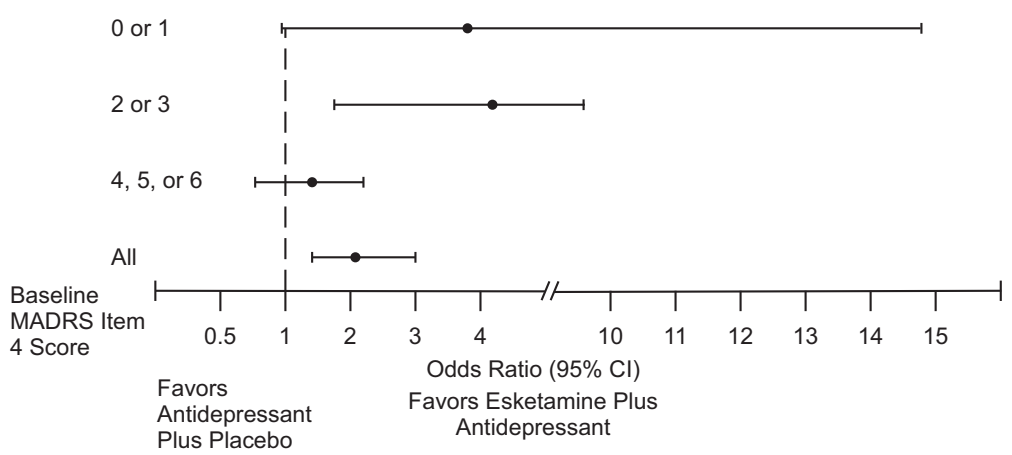

B Remission

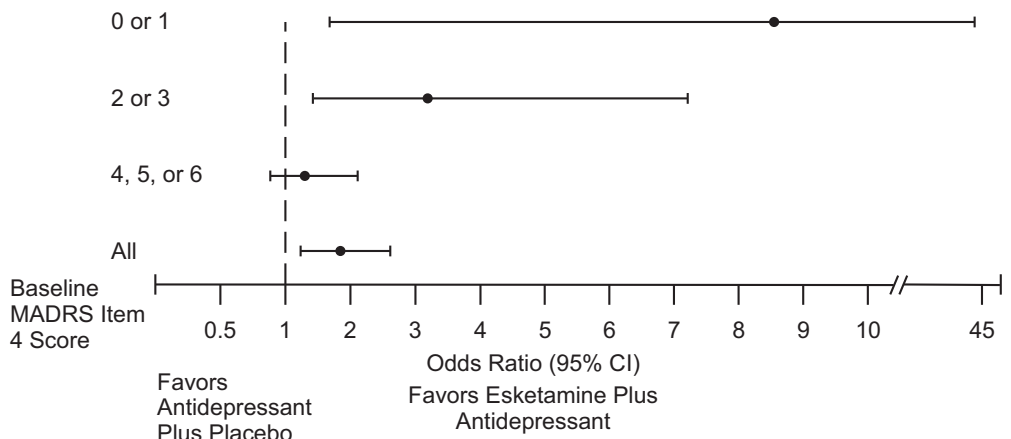

Esketamine Plus Antidepressant Plus

Antidepressant

\begin{tabular}{cccc} 
N & Proportion & N & Proportion \\
\hline $16 / 31$ & $51.6 \%$ & $6 / 20$ & $30.0 \%$
\end{tabular}

$42 / 69$

$60.9 \%$

$18 / 58$

$31.0 \%$

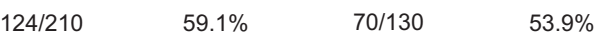

$\begin{array}{llll}182 / 310 & 58.7 \% & 94 / 208 & 45.2 \%\end{array}$

Figure 2 Response (A) and Remission (B) Rates at Day 28 Based on Montgomery-Åsberg Depression Rating Scale Total Score.

Notes: Response was defined as a $\geq 50 \%$ improvement in MADRS total score from baseline. Remission was defined as MADRS total score $\leq 12$.

Abbreviations: $\mathrm{Cl}$, confidence interval; MADRS, Montgomery-Åsberg Depression Rating Scale.

$P=0.004)$; this improvement increased over the doubleblind treatment period $(49.0 \%$ [152/310] and $41.4 \%$, [86/208], respectively, $P=0.038$, at day 28).

Among the patients with the most severe sleep disturbance at baseline (scores of 4, 5, or 6), 42.5\% (91/214) of patients in the esketamine plus antidepressant group and $28.7 \%(37 / 129)$ patients in the antidepressant plus placebo group reported less sleep disturbance (score of $0,1,2$, or 3) at 24 hours-post first dose. The improvement seen after a single night of sleep was sustained over the course of the 4 weeks of treatment, with $62.4 \%$ (131/210) and $57.7 \%(75 / 130)$ of patients in the respective treatment groups reporting amelioration of sleep disturbance from moderate-to-severe at baseline to a lower level at day 28 . At day 28, less than a third of esketamine-treated remitters $(32.8 \%, 43 / 131)$ and placebo-treated remitters $(32.8 \%, 21 /$ 64) had a MADRS item 4 score $>1$ at day 28.

\section{Is early change in reported sleep disturbance severity related to likelihood of response or remission of depressive symptoms at day 28 ?}

The more sleep improved over the first week of treatment, the higher the likelihood of antidepressant response or remission at day 28: across both the esketamine and placebo groups, a 1-point improvement in sleep at day 8 was associated with a $26 \%$ increased probability of antidepressant response $(\mathrm{OR}=1.26,95 \% \mathrm{CI}: 1.12,1.42 ; P<0.001)$ and a $28 \%$ increased probability of remission $(\mathrm{OR}=1.28$, 95\% CI: $1.14,1.43 ; P<0.001)$. The association between early improvement in sleep disturbance and response at day 28 (interaction $P=0.530$ ) and remission at day 28 (interaction $P=0.292$ ) did not differ between the treatment groups. Among patients in the esketamine plus 


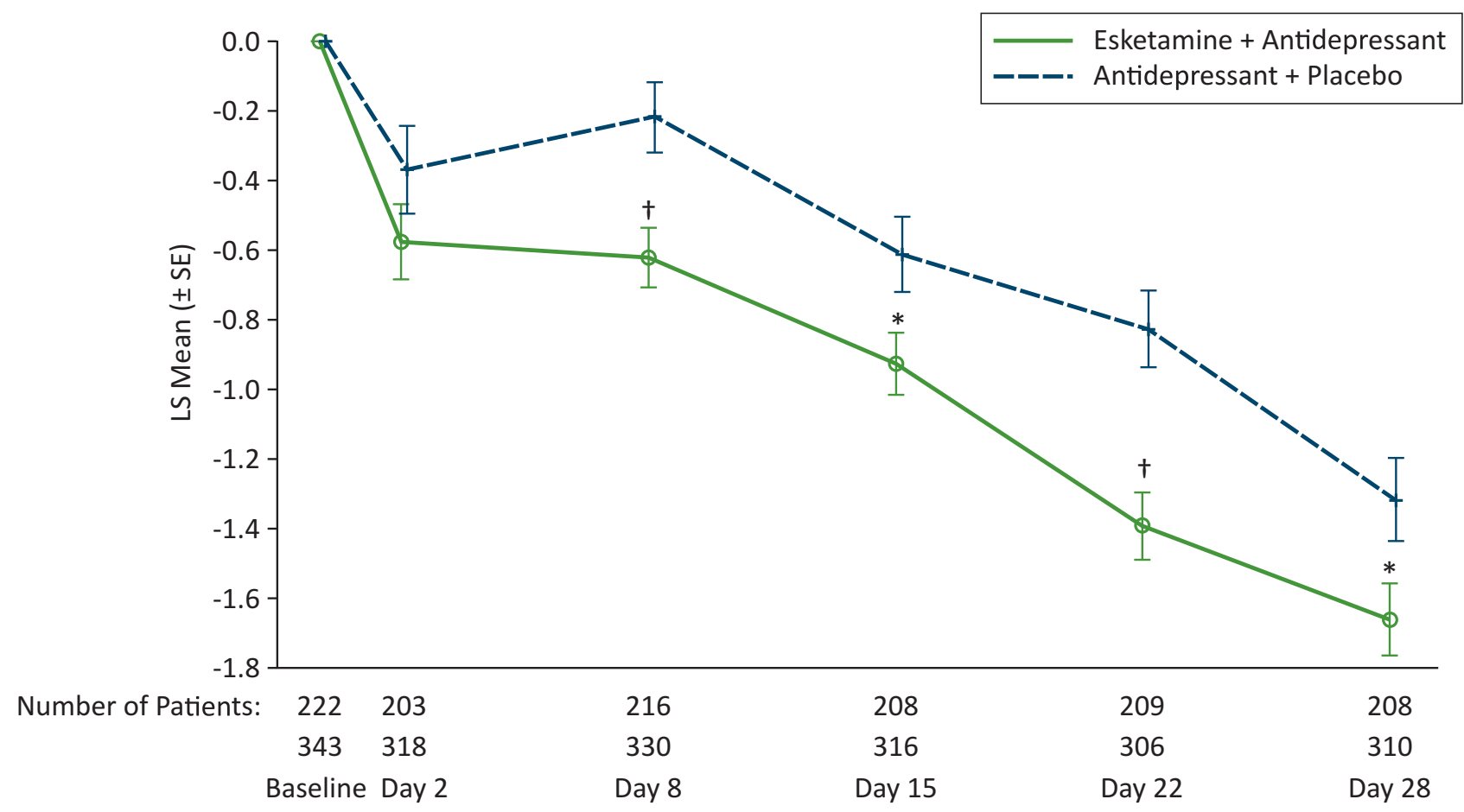

Figure 3 Least Square Mean Change (SE) in Montgomery-Åsberg Depression Rating Scale Item 4 Score Over Time in the Double-Blind Treatment Phase. Notes: $* P \leq 0.02,{ }^{\dagger} P \leq 0.00$; LS mean difference between groups from ANCOVA model with fixed effects for treatment, region, class of oral antidepressant (SNRI or SSRI), study ID and baseline value as a covariate. Analysis is based on change from baseline.

Abbreviations: LS, least squares; MADRS, Montgomery-Åsberg Depression Rating Scale; SE, standard error.

antidepressant group, early improvement in sleep was associated with a $20 \%$ increased probability of antidepressant response $(\mathrm{OR}=1.20,95 \% \mathrm{CI}: 1.05,1.39 ; P=0.009)$ and of remission $(\mathrm{OR}=1.20,95 \% \mathrm{CI}$ : 1.04, 1.37; $P=0.011)$. Among patients in the antidepressant plus placebo group, early improvement in sleep was associated with a $30 \%$ increased probability of antidepressant response $(\mathrm{OR}=1.30,95 \% \mathrm{CI}: 1.06,1.61 ; P=0.011)$ and a $37 \%$ increased probability of remission $(\mathrm{OR}=1.37,95 \%$ CI: $1.10,1.72 ; P=0.006)$.

\section{Safety Findings}

Adverse events are summarized by severity of sleep disturbance at baseline in Table 2. The most common adverse events reported for esketamine-treated patients $(>10 \%$ of all patients) were dissociation, dizziness, dysgeusia, headache, hypoesthesia, hypoesthesia oral, nausea, paresthesia, somnolence, and vertigo (Table 2). The discontinuation rate due to adverse events was low (1.4\% to $6.3 \%)$ across all the baseline sleep disturbance status and treatment subgroups (Table 2).

\section{Discussion}

Given that disturbed sleep is one of nine diagnostic criteria for MDD, it stands to reason that sleep disturbance is a commonly occurring condition in MDD. ${ }^{35}$ In a US epidemiological study of patients with MDD, most reported at least one type of sleep complaint. $^{36}$ In the TRANSFORM studies, approximately two-thirds of the patients with TRD presented with moderate or severe sleep disturbance (defined by item 4 of the MADRS score $\geq 4$ ), in line with results from other studies measuring sleep disturbance via the sleep item of a depression severity rating scale. ${ }^{37}$

Severity of patient-reported sleep disturbance has been correlated with overall depression severity and poorer quality of life. ${ }^{38}$ In our sample of patients with TRD, mean MADRS total- excluding item 4 - score was higher in the subgroups with greater sleep disturbance at baseline. Insomnia and poor sleep have been associated with increased risk for depression, ${ }^{39}$ depression severity, risk of relapse, as well as increased risk for suicidal ideation and attempt, ${ }^{10,40}$ raising the question of the importance of treating sleep disturbance during MDD treatment. ${ }^{14,41}$

Persistently disturbed sleep after successful treatment of depression increases the risk of depression recurrence. ${ }^{42}$ Even after achieving remission of their depression, a substantial percentage of patients treated with standard oral antidepressants continue to have residual sleep 


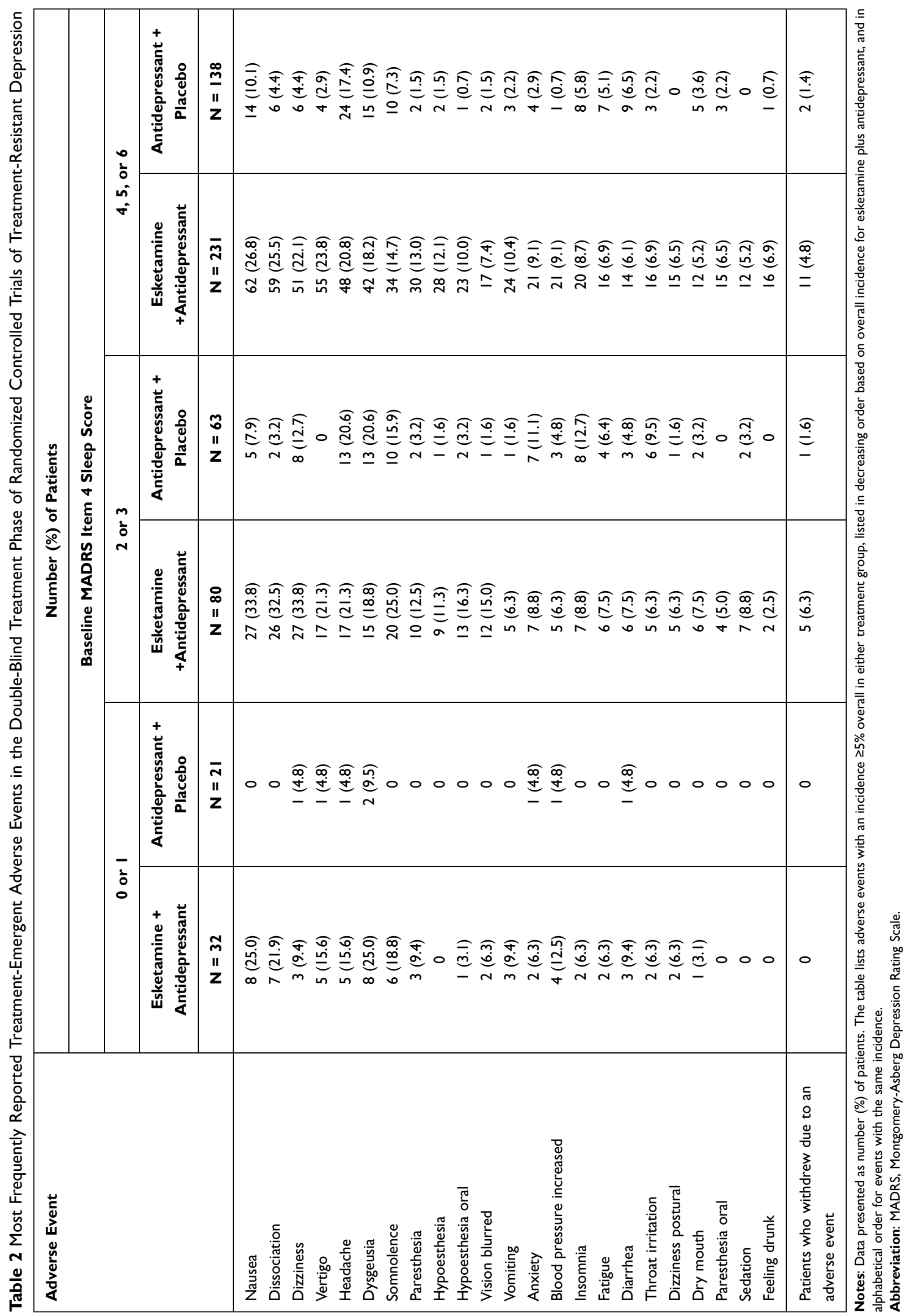


disturbance $^{43}$ (eg, $>40 \%$ with fluoxetine ${ }^{44}$ ), suggesting that the management of depression can be improved by specifically targeting sleep disturbance in addition to the overall depression. In the TRANSFORM studies, sleep improved after the first dose of study drug, with between-group separation favoring esketamine beginning after 1 week of treatment and persisting thereafter through the entire month of double-blind treatment. Less than a third of esketamine-treated patients continued to have some degree of sleep disturbance after achieving remission at day 28 in the TRANSFORM studies. Sleep score improved by $30 \%$ with antidepressant (SSRI/SNRI) plus placebo and by $38 \%$ with esketamine plus antidepressant after 4 weeks of treatment in our analysis. By way of comparison, assessing 2788 adults with MDD and insomnia who participated in the STAR*D trial, Mason et al found that insomnia symptoms improved by $29 \%$ during the acute phase (up to 14 weeks) of antidepressant treatment. ${ }^{19}$

Sleep disturbance has been associated with poor response to psychotherapy and pharmacologic treatment of depression in some studies, while other studies did not demonstrate that baseline insomnia severity impacts monoaminergic antidepressant efficacy. ${ }^{43}$ On the basis of these divergent findings, we investigated whether baseline sleep disturbance influences overall antidepressant efficacy in patients with TRD. Overall, the greater the severity of sleep disturbance at baseline, the greater the likelihood that depressive symptoms would respond or remit by day 28 , similar to an observation made by another research group following intravenous ketamine treatment of patients with unipolar and bipolar depression. $^{21}$ In a comparison of treatment groups, we observed a higher antidepressant response rate $(\mathrm{OR}=2.05)$ and remission rate $(\mathrm{OR}=1.81)$ with esketamine plus antidepressant than with antidepressant plus placebo, regardless of the presence of sleep disturbance at baseline. A definitive explanation for this finding remains to be determined. In our study, a relationship between early improvement in sleep and increased probability of response and remission of depressive symptoms was observed in both treatment groups.

Several studies of patients with MDD and sleep disturbance found that concomitant treatment of sleep disturbance and depression enhances depression outcome compared with treatment of only the depressive disorder. $^{45,46}$ Confirming this association, we observed that the more sleep improved over the first week of treatment, the greater the likelihood of response and of remission at day 28. Early improvement in sleep with esketamine, leading to improved depression outcomes, may encourage patients with TRD to complete at least 4 weeks of treatment to determine efficacy.

The safety of esketamine nasal spray for patients with TRD was rigorously evaluated in the TRANSFORM studies and reported in detail elsewhere. ${ }^{24,25}$ In this work, the adverse event profile of esketamine, based on sleep disturbance category, was generally similar to that observed overall in the primary studies. Few esketamine-treated patients discontinued prematurely, irrespective of sleep disturbance severity.

The impact that sleep disturbance has on depression, and vice versa, underscores the need for optimal treatment of both. Depression accompanied by sleep disturbance are oftentimes simultaneously treated, with one or both too frequently inadequately treated. The beneficial effect of esketamine on sleep disturbance is a clinical advantage not always seen with SSRIs and SNRIs.

\section{Study Limitations}

Our results are based on exploratory, post-hoc analyses of MADRS item 4 score, with the limitations of post hoc analyses acknowledged. ${ }^{47}$ Other standard clinician-reported instruments to assess sleep disturbance and patient-reported assessment of sleep were not performed in the TRANSFORM studies, which could have added to the interpretation of our findings. Moreover, the generalizability of our analyses must consider the exclusion of patients $\geq 65$ years old, those with moderate-to-severe substance and alcohol use disorder, and those taking high-dose benzodiazepines. Finally, this study did not include objective indicators of sleep such as actigraphy and polysomnography. In this regard, favorable antidepressant response is accompanied by improvement in slow wave sleep in both acute and maintenance trials. ${ }^{48,49}$ And, in MDD, ketamine's rapid antidepressant effects have been linked to increased total sleep, slow wave sleep, slow wave activity and rapid eye movement sleep as well as to decreased waking. ${ }^{50}$

\section{Conclusions}

Exploratory, post-hoc analyses support the antidepressant efficacy of esketamine nasal spray in patients with TRD, regardless of the presence or absence of sleep disturbance. One week after the initiation of treatment, more esketamine-treated patients reported clinically meaningful improvement in sleep than those taking oral antidepressant plus placebo. Additional research, using objective markers of sleep and established clinical assessment tools of insomnia, will expand upon these findings. 


\section{Abbreviations}

ANCOVA, analysis of covariance; CI, confidence interval; DSM-5, Diagnostic and Statistical Manual of Mental Disorders 5th edition; LOCF, last observation carried forward; LS, least-squares; MADRS, Montgomery Åsberg Depression Rating Scale; MDD, major depressive disorder; OR, odds ratio; SD, standard deviation; SE, standard error; SNRI, serotonin norepinephrine reuptake inhibitor; SSRI, selective serotonin reuptake inhibitor; STAR*D, Sequenced Treatment Alternatives to Relieve Depression; TRD, treatment-resistant depression.

\section{Acknowledgments}

We acknowledge Sandra Norris, PharmD of the Norris Communications Group LLC, supported by Janssen Research \& Development, LLC, who provided medical writing assistance and Ellen Baum, PhD (Janssen Global Services, LLC) who provided additional editorial support and was not compensated.

\section{Author Contributions}

Dr. Ibrahim Turkoz conducted the statistical analyses. All authors made a significant contribution to the work reported, whether that is in the conception, study design, execution, acquisition of data, analysis and interpretation, or in all these areas; took part in drafting, revising or critically reviewing the article; gave final approval of the version to be published; have agreed on the journal to which the article has been submitted; and agree to be accountable for all aspects of the work. All authors meet ICMJE criteria and all those who fulfilled those criteria are listed as authors.

\section{Funding}

The TRANSFORM-1 and TRANSFORM-2 studies were funded by Janssen Research \& Development, LLC, Titusville, NJ, USA.

\section{Disclosure}

Drs. Stephane Borentain, David Williamson, Ibrahim Turkoz, Vanina Popova, and Maju Mathews are employees of Janssen Research \& Development, LLC, Janssen Scientific Affairs, LLC or Janssen Research \& Development, Belgium, and some are stockholders of Johnson \& Johnson, Inc. Dr. Frank Wiegand was an employee of Janssen Research \& Development, LLC at the time the study was performed and is now employed by Beckley Psytech Ltd. Dr. W Vaughan McCall receives honoraria from Wolters Kluwer Publishing, CME Outfitters, and Anthem Inc., and research support from Merck and MECTA Corp, and is a scientific advisor for Sage Therapeutics, Janssen, Jazz, Eisai, and Idorsia. The authors report no other conflicts of interest in this work.

\section{References}

1. Sunderajan P, Gaynes BN, Wisniewski SR, et al. Insomnia in patients with depression: a STAR*D report. CNS Spectr. 2010;15(6):394-404. doi:10.1017/S1092852900029266

2. American Psychiatric Association. Diagnostic and Statistical Manual of Mental Disorders, 5th Edition (DSM-5). Arlington, TX: American Psychiatric Publishing; 2013.

3. Baglioni C, Battagliese G, Feige B, et al. Insomnia as a predictor of depression: a meta-analytic evaluation of longitudinal epidemiological studies. J Affect Disord. 2011;135(1-3):10-19. doi:10.1016/j. jad.2011.01.011

4. Fang H, Tu S, Sheng J, Shao A. Depression in sleep disturbance: a review on a bidirectional relationship, mechanisms and treatment. J Cell Mol Med. 2019;23(4):2324-2332. doi:10.1111/jcmm.14170

5. Cepeda MS, Reps J, Ryan P. Finding factors that predict treatment-resistant depression: results of a cohort study. Depress Anxiety. 2018;35(7):668-673. doi:10.1002/da.22774

6. Franzen PL, Buysse DJ. Sleep disturbances and depression: risk relationships for subsequent depression and therapeutic implications. Dialogues Clin Neurosci. 2008;10(4):473-481. doi:10.31887/DCNS.2008.10.4/plfranzen

7. McCall WV, Blocker JN, D’Agostino R, et al. Insomnia severity is an indicator of suicidal ideation during a depression clinical trial. Sleep Med. 2010;11(9):822-827. doi:10.1016/j.sleep.2010.04.004

8. Ohayon MM, Roth T. Place of chronic insomnia in the course of depressive and anxiety disorders. J Psychiatr Res. 2003;37(1):9-15. doi:10.1016/s0022-3956(02)00052-3

9. Ohayon MM. Observation of the natural evolution of insomnia in the American general population cohort. Sleep Med Clin. 2009;4 (1):87-92. doi:10.1016/j.jsmc.2008.12.002

10. Pigeon WR, Pinquart M, Conner K. Meta-analysis of sleep disturbance and suicidal thoughts and behaviors. J Clin Psychiatry. 2012;73(9):e1160-e1167. doi:10.4088/JCP.11r07586

11. van Mill JG, Vogelzangs N, Hoogendijk WJ, Penninx BW. Sleep disturbances and reduced work functioning in depressive or anxiety disorders. Sleep Med. 2013;14(11):1170-1177. doi:10.1016/j. sleep.2013.04.016

12. McCall WV, Blocker JN, D’Agostino R, et al. Treatment of insomnia in depressed insomniacs: effects on health-related quality of life, objective and self-reported sleep, and depression. J Clin Sleep Med. 2010;6(4):322-329. doi:10.5664/jcsm. 27872

13. McCall WV, Benca RM, Rosenquist PB, et al. Reducing suicidal ideation through insomnia treatment (REST-IT): a randomized clinical trial. Am J Psychiatry. 2019;176(11):957-965. doi:10.1176/appi. ajp.2019.19030267

14. Gebara MA, Siripong N, DiNapoli EA, et al. Effect of insomnia treatments on depression: a systematic review and meta-analysis. Depress Anxiety. 2018;35(8):717-731. doi:10.1002/da.22776

15. Bei B, Asarnow LD, Krystal A, Edinger JD, Buysse DJ, Manber R. Treating insomnia in depression: insomnia related factors predict long-term depression trajectories. J Consult Clin Psychol. 2018;86 (3):282-293. doi:10.1037/ccp0000282

16. Pigeon WR, Hegel M, Unützer J, et al. Is insomnia a perpetuating factor for late-life depression in the IMPACT cohort? Sleep. 2008;31 (4):481-488. doi:10.1093/sleep/31.4.481 
17. Troxel WM, Kupfer DJ, Reynolds CF, et al. Insomnia and objectively measured sleep disturbances predict treatment outcome in depressed patients treated with psychotherapy or psychotherapy-pharmacotherapy combinations. J Clin Psychiatry. 2012;73(4):478-485. doi:10.4088/ JCP. $11 \mathrm{~m} 07184$

18. Wade AG. Sleep problems in depression: how do they impact treatment and recovery? Int J Psychiatry Clin Pract. 2006;10(Suppl 1):38-44. doi:10.1080/13651500600552594

19. Mason BL, Davidov A, Minhajuddin A, Trivedi MH. Focusing on insomnia symptoms to better understand depression: a STAR*D report. $J$ Affect Disord. 2020;260:183-186. doi:10.1016/j.jad.2019.08.094

20. Fava M, Hoog SL, Judge RA, Kopp JB, Nilsson ME, Gonzales JS. Acute efficacy of fluoxetine versus sertraline and paroxetine in major depressive disorder including effects of baseline insomnia. J Clin Psychopharmacol. 2002;22(2):137-147. doi:10.1097/00004714200204000-00006

21. Liu W, Zhou Y, Wang C, et al. Baseline insomnia as a predictor of antidepressant efficacy to repeated intravenous ketamine for unipolar and bipolar depression: a preliminary study. $J$ Affect Disord. 2020;271:1-8. doi:10.1016/j.jad.2020.03.048

22. Janssen Pharmaceutical Companies. Spravato ${ }^{T M}$ (Esketamine) Nasal Spray Prescribing Information. (C). Janssen Pharmaceutical Companies; 2020.

23. Spravato (esketamine) summary of product characteristics. Available from: https://www.medicines.org.uk/emc/product/10977/smpc. Accessed August 22, 2021.

24. Fedgchin M, Trivedi M, Daly EJ, et al. Efficacy and safety of fixed-dose esketamine nasal spray combined with a new oral antidepressant in treatment-resistant depression: results of a randomized, double-blind, active-controlled study (TRANSFORM-1). Int J Neuropsychopharmacol. 2019;22(10):616-630. doi:10.1093/ ijnp/pyz039

25. Popova V, Daly EJ, Trivedi M, et al. Efficacy and safety of flexibly dosed esketamine nasal spray combined with a newly initiated oral antidepressant in treatment-resistant depression: a randomized double-blind active-controlled study. Am J Psychiatry. 2019;176 (6):428-438. doi:10.1176/appi.ajp.2019.19020172

26. Trivedi MH, Rush AJ, Ibrahim HM, et al. The Inventory of Depressive Symptomatology, Clinician Rating (IDS-C) and Self-Report (IDS-SR), and the Quick Inventory of Depressive Symptomatology, Clinician Rating (QIDS-C) and Self-Report (QIDS-SR) in public sector patients with mood disorders: a psychometric evaluation. Psychol Med. 2004;34(1):73-82. doi:10.1017/s0033291703001107

27. Montgomery SA, Asberg M. A new depression scale designed to be sensitive to change. $B r \quad J$ Psychiatry. 1979;134(4):382-389. doi:10.1192/bjp.134.4.382

28. Williams JBW, Kobak KA. Development and reliability of a structured interview guide for the Montgomery-Asberg Depression Rating Scale (SIGMA). Br J Psychiatry. 2008;192 (1):52-58. doi:10.1192/bjp.bp.106.032532

29. Bertschy G, Ragama-Pardos E, Muscionico M, et al. Trazodone addition for insomnia in venlafaxine-treated, depressed inpatients: a semi-naturalistic study. Pharmacol Res. 2005;51(1):79-84. doi:10.1016/j.phrs.2004.06.007

30. Gebara MA, Kasckow J, Smagula SF, et al. The role of late life depressive symptoms on the trajectories of insomnia symptoms during antidepressant treatment. J Psychiatr Res. 2018;96:162-166. doi:10.1016/j.jpsychires.2017.10.013

31. Lader M, Andersen HF, Baekdal T. The effect of escitalopram on sleep problems in depressed patients. Human Psychopharmacol. 2005;20(5):349-354. doi:10.1002/hup.694

32. Schweitzer I, Burrows G, Tuckwell V, et al. Sustained response to open-label venlafaxine in drug-resistant major depression. J Clin Psychopharmacol. 2001;21(2):185-189. doi:10.1097/00004714200104000-00010
33. Stein DJ, Lopez AG. Effects of escitalopram on sleep problems in patients with major depression or generalized anxiety disorder. $A d v$ Ther. 2011;28(11):1021-1037. doi:10.1007/s12325-011-0071-8

34. Trivedi MH, Bandelow B, Demyttenaere K, et al. Evaluation of the effects of extended release quetiapine fumarate monotherapy on sleep disturbance in patients with major depressive disorder: a pooled analysis of four randomized acute studies. Int J Neuropsychopharmacol. 2013;16(8):1733-1744. doi:10.1017/ S146114571300028X

35. Ohayon MM. Prevalence of DSM-IV diagnostic criteria of insomnia: distinguishing insomnia related to mental disorders from sleep disorders. J Psychiatr Res. 1997;31:333-346. doi:10.1016/s00223956(97)00002-2

36. Geoffroy PA, Hoertel N, Etain B, et al. Insomnia and hypersomnia in major depressive episode: prevalence, sociodemographic characteristics and psychiatric comorbidity in a population-based study. J Affective Disord. 2018;226:132-141. doi:10.1016/j.jad.2017.09.032

37. Perlis ML, Giles DE, Buysse DJ, Thase ME, Tu X, Kupfer DJ. Which depressive symptoms are related to which sleep electroencephalographic variables? Biol Psychiatry. 1997;42(10):904-913. doi:10.1016/S0006-3223(96)00439-8

38. McCall WV, Reboussin BA, Cohen W. Subjective measurement of insomnia and quality of life in depressed inpatients. J Sleep Res. 2000;9(1):43-48. doi:10.1046/j.1365-2869.2000.00186.x

39. Ford DE, Kamerow DB. Epidemiologic study of sleep disturbances and psychiatric disorders. An opportunity for prevention? JAMA. 1989;262(11):1479-1484. doi:10.1001/jama.262.11.1479

40. Chellappa SL, Araújo JF. Sleep disorders and suicidal ideation in patients with depressive disorder. Psychiatry Res. 2007;153 (2):131-136. doi:10.1016/j.psychres.2006.05.007

41. Bishop TM, Walsh PG, Ashrafioun L, Lavigne JE, Pigeon WR. Sleep, suicide behaviors, and the protective role of sleep medicine. Sleep Med. 2020;66:264-270. doi:10.1016/j.sleep.2019.07.016

42. Dombrovski AY, Mulsant BH, Houck PR, et al. Residual symptoms and recurrence during maintenance treatment of late-life depression. J Affect Disord. 2007;103(1-3):77-82. doi:10.1016/j.jad.2007.01.020

43. Trivedi MH, Hollander E, Nutt D, Blier P. Clinical evidence and potential neurobiological underpinnings of unresolved symptoms of depression. J Clin Psychiatry. 2008;69(2):246-258. doi:10.4088/jcp. v69n0211

44. Nierenberg AA, Keefe BR, Leslie VC, et al. Residual symptoms in depressed patients who respond acutely to fluoxetine. J Clin Psychiatry. 1999;60(4):221-225. doi:10.4088/jcp.v60n0403

45. Fava M, McCall WV, Krystal A, et al. Eszopiclone co-administered with fluoxetine in patients with insomnia coexisting with major depressive disorder. Biol Psychiatry. 2006;59(11):1052-1060. doi:10.1016/j.biopsych.2006.01.016

46. Manber R, Edinger JD, Gress JL, San Pedro-Salcedo MG, Kuo TF, Kalista T. Cognitive behavioral therapy for insomnia enhances depression outcome in patients with comorbid major depressive disorder and insomnia. Sleep. 2008;31(4):489-495. doi:10.1093/sleep/ 31.4.489

47. Curran-Everett D, Milgrom H. Post-hoc data analysis: benefits and limitations. Curr Opin Allergy Clin Immunol. 2013;13(3):223-224. doi:10.1097/ACI.0b013e3283609831

48. Kupfer DJ, Ehlers CL, Frank E, Grochocinski VJ, McEachran AB, Buhari A. Persistent effects of antidepressants: EEG sleep studies in depressed patients during maintenance treatment. Biol Psychiatry. 1994;35(10):781-793. doi:10.1016/0006-3223(94)91140-1

49. Reynolds CF, Hoch CC, Buysse DJ, et al. Sleep in late-life recurrent depression. Changes during early continuation therapy with nortriptyline. Neuropsychopharmacol. 1991;5(2):85-96.

50. Duncan WC, Sarasso S, Ferrarelli F, et al. Concomitant BDNF and sleep slow wave changes indicate ketamine-induced plasticity in major depressive disorder. Int J Neuropsychopharmacol. 2013;16 (2):301-311. doi:10.1017/S1461145712000545 


\section{Publish your work in this journal}

Neuropsychiatric Disease and Treatment is an international, peerreviewed journal of clinical therapeutics and pharmacology focusing on concise rapid reporting of clinical or pre-clinical studies on a range of neuropsychiatric and neurological disorders. This journal is indexed on PubMed Central, the 'PsycINFO' database and CAS, and is the official journal of The International Neuropsychiatric Association (INA). The manuscript management system is completely online and includes a very quick and fair peer-review system which is all easy to use. Visit http://www.dovepress.com/testimonials.php to read real quotes from published authors.

Submit your manuscript here: https://www.dovepress.com/neuropsychiatric-disease-and-treatment-journal 